\title{
Schottky barrier tuning via dopant segregation in NiGeSn-GeSn contacts
}

Christian Schulte-Braucks, Emily Hofmann, Stefan Glass, Nils von den Driesch, Gregor Mussler, Uwe Breuer, Jean-Michel Hartmann, Peter Zaumseil, Thomas Schröder, Qing-Tai Zhao, Siegfried Mantl, and Dan Buca

Citation: Journal of Applied Physics 121, 205705 (2017); doi: 10.1063/1.4984117

View online: http://dx.doi.org/10.1063/1.4984117

View Table of Contents: http://aip.scitation.org/toc/jap/121/20

Published by the American Institute of Physics

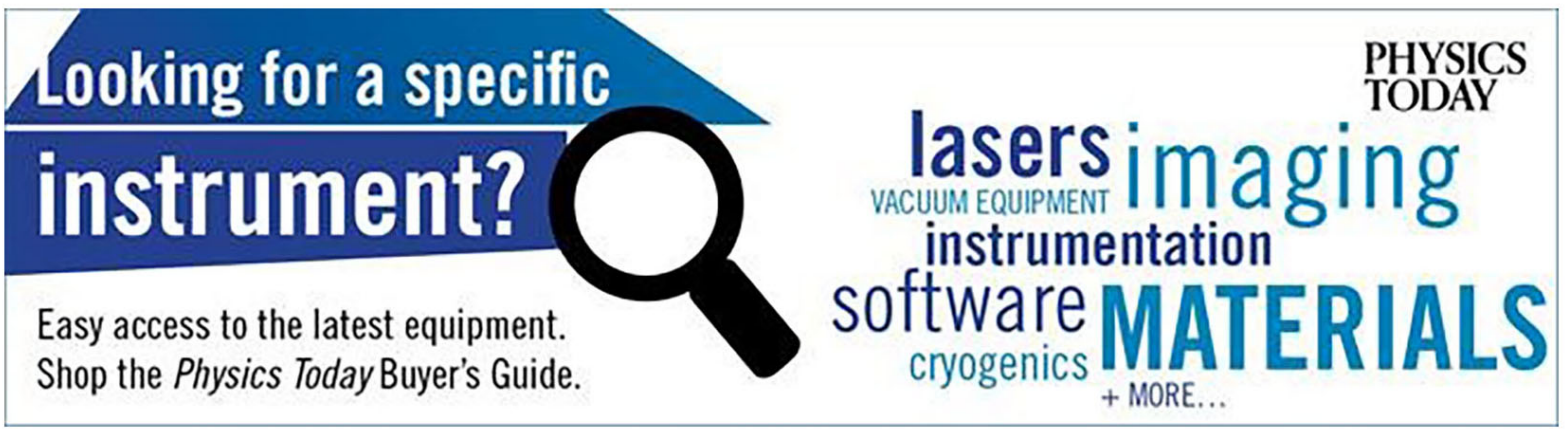




\title{
Schottky barrier tuning via dopant segregation in NiGeSn-GeSn contacts
}

\author{
Christian Schulte-Braucks, ${ }^{1}$ Emily Hofmann, ${ }^{1}$ Stefan Glass, ${ }^{1}$ Nils von den Driesch, ${ }^{1}$ \\ Gregor Mussler, ${ }^{1}$ Uwe Breuer, ${ }^{2}$ Jean-Michel Hartmann, ${ }^{3}$ Peter Zaumseil, ${ }^{4}$ \\ Thomas Schröder, ${ }^{4,5}$ Qing-Tai Zhao, ${ }^{1}$ Siegfried Mantl, ${ }^{1}$ and Dan Buca ${ }^{1}$ \\ ${ }^{1}$ Peter Grünberg Institut (PGI 9), Forschungszentrum Jülich GmbH, Jülich 52428, Germany \\ ${ }^{2}$ Zentralinstitut für Engineering, Elektronik und Analytik (ZEA-3), Forschungszentrum Jülich GmbH, \\ 52428 Jülich, Germany \\ ${ }^{3}$ University of Grenoble Alpes and CEA, LETI, MINATEC Campus, F-38000 Grenoble, France \\ ${ }^{4}$ IHP, Im Technologiepark 25, 15236 Frankfurt (Oder), Germany \\ ${ }^{5}$ Brandenburgisch Technische Universität BTU, Institut für Physik, Konrad Zuse Str.1, 03046 Cottbus, \\ Germany
}

(Received 3 March 2017; accepted 12 May 2017; published online 31 May 2017)

\begin{abstract}
We present a comprehensive study on the formation and tuning of the Schottky barrier of NiGeSn metallic alloys on $\mathrm{Ge}_{1-\mathrm{x}} \mathrm{Sn}_{\mathrm{x}}$ semiconductors. First, the Ni metallization of GeSn is investigated for a wide range of $\mathrm{Sn}$ contents $(\mathrm{x}=0-0.125)$. Structural analysis reveals the existence of different poly-crystalline $\mathrm{NiGeSn}$ and $\mathrm{Ni}_{3}(\mathrm{GeSn})_{5}$ phases depending on the $\mathrm{Sn}$ content. Electrical measurements confirm a low NiGeSn sheet resistance of $12 \Omega / \square$ almost independent of the Sn content. We extracted from Schottky barrier height measurements in NiGeSn/GeSn/NiGeSn metal-semiconductor-metal diodes Schottky barriers for the holes below $0.15 \mathrm{eV}$. They decrease with the Sn content, thereby confirming NiGeSn as an ideal metal alloy for p-type contacts. Dopant segregation for both p- and n-type dopants is investigated as a technique to effectively modify the Schottky barrier of $\mathrm{NiGeSn/GeSn} \mathrm{contacts.} \mathrm{Secondary} \mathrm{ion} \mathrm{mass} \mathrm{spectroscopy} \mathrm{is} \mathrm{employed} \mathrm{to} \mathrm{analyze} \mathrm{dopant} \mathrm{segrega-}$ tion and reveal its dependence on both the Sn content and biaxial layer strain. Published by AIP Publishing. [http://dx.doi.org/10.1063/1.4984117]
\end{abstract}

\section{INTRODUCTION}

Germanium tin (GeSn) alloys have been the subject of numerous advances in recent years, as highlighted in Ref. 1. Substantial improvements in epitaxial growth have led in 2015 to the first demonstration of lasing in a group IV alloy. ${ }^{2,3}$ Furthermore, the fabrication of GeSn based electronic devices such as Metal Oxide Semiconductor Field Effect Transistors (MOSFETs), ${ }^{4,5}$ Tunnel FETs (TFETs), ${ }^{6-8}$ and tunnel-diodes ${ }^{9}$ has qualified GeSn as an interesting material in order to (i) boost the mobility in the channels of MOSFETs and (ii) obtain a direct band-to-band tunneling in TFETs. However, in order to assess the real potential of a new semiconductor alloy, device process modules and fabrication technologies also need to be studied and optimized. Due to the reduced thermal stability of high Sn content GeSn, temperatures above $350^{\circ} \mathrm{C}$ have to be avoided to prevent Sn-diffusion and precipitation. The availability of lowresistive contacts is a key module to fabricate high performance electronics and opto-electronics devices. This work provides a comprehensive study on the formation and characterization of $\mathrm{NiGeSn} / \mathrm{GeSn}$-contacts including the redistribution of dopants during NiGeSn formation.

The formation of metal-semiconductor alloys (such as silicides or germanides) is a well-established method to obtain smooth and low resistive contacts on semiconductors. The Schottky barrier formed at the metal-semiconductor interface should be as low as possible for realizing good Ohmic characteristics. Dopant segregation (DS) can, beside the proper selection of the alloying metal itself, be used to tune the Schottky barrier height $(\mathrm{SBH}){ }^{10}$ The aim is then to achieve a high doping concentration at the metal semiconductor junction, which induces a strong electronic band bending and in turn modifies the effective Schottky barrier height, yielding a reduced contact resistivity. Two methods are mainly used to perform dopant segregation (DS): (i) first, the metallic alloy (e.g., silicide) is formed followed by dopant implantation into the alloy [e.g., implantation-intosilicide (IIS)]. Then, the dopants are driven towards the alloy-semiconductor interface in a second annealing step; (ii) in the alternative approach, dopants are implanted into the semiconductor prior to the metal deposition. The metallic alloy formation and the dopant segregation then occur during the subsequent annealing as one step. In both schemes, the implantation energy is chosen such that the ion implanted region lies completely within the metal alloy or is fully consumed by the subsequent metallization. Mainly metal-Si alloys (i.e., silicides like $\mathrm{NiSi}, \mathrm{NiAlSi}$, and $\mathrm{CoSi}_{2}$ ) but also metal-Ge alloys (i.e., germanides such as PtGe and $\mathrm{NiGe)}$ have been widely studied for different metals and doping species. ${ }^{11,12}$ However, for Sn-based alloys there are only a few studies on nickel-stano-germanide $\left(\mathrm{NiGeSn}^{13-17}\right)$ and NiPtGeSn. ${ }^{18}$ In those studies, Schottky barriers were modified with dopants such as boron atoms ${ }^{15}$ or impurities such as sulfur and selenium atoms. ${ }^{16}$

In this paper, we present a comprehensive study of $\mathrm{NiGeSn}$ contact formation including $\mathrm{B}, \mathrm{P}$, and As dopant segregation. A systematic structural analysis was performed using transmission-electron-microscopy (TEM) and X-ray diffraction (XRD) followed by electrical characterization. We evaluated the sheet resistance of NiGeSn, as well as the 

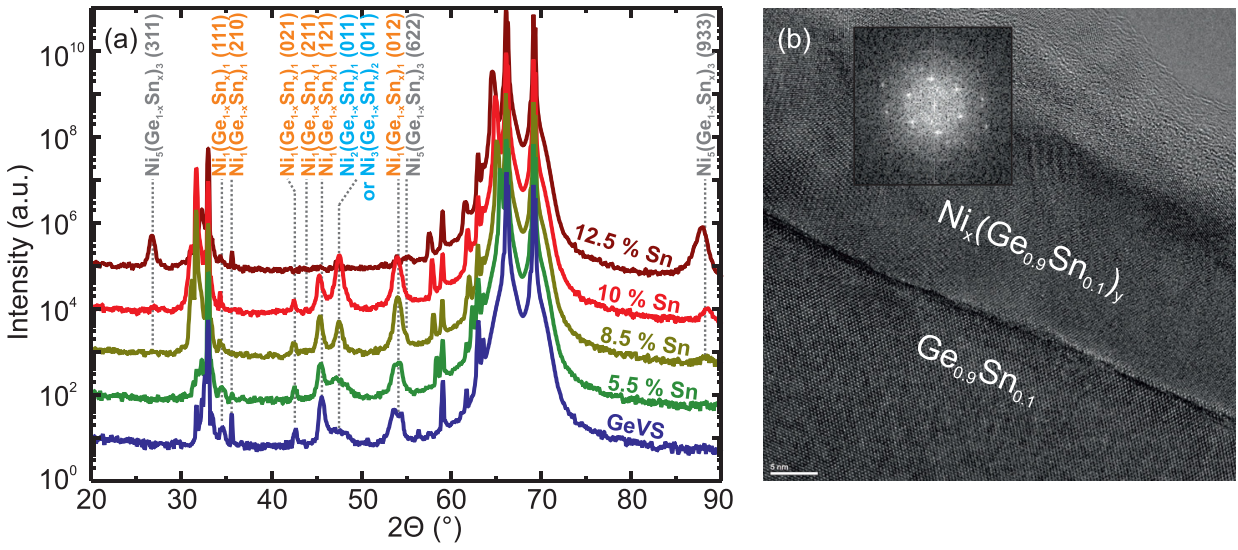

FIG. 1. (a) XRD $\theta-2 \theta$ spectra of $\mathrm{Ni}_{\mathrm{y}}\left(\mathrm{Ge}_{\mathrm{x}} \mathrm{Sn}_{1-\mathrm{x}}\right)_{\mathrm{z}}$. The unlabeled peaks belong to reflections from $\mathrm{GeSn}$, GeVS, and $\mathrm{Si}$ substrates. (b) XTEM image of $\mathrm{Ni}\left(\mathrm{Ge}_{0.9} \mathrm{Sn}_{0.1}\right)$ showing the presence of a smooth interface with the $\mathrm{Ge}_{0.9} \mathrm{Sn}_{0.1}$ layer underneath. The inset shows the FFT pattern of the NiGeSn layer, with a hexagonal symmetry evidenced. contact resistance and Schottky barrier height (SBH) of $\mathrm{NiGeSn/GeSn} \mathrm{systems.} \mathrm{Emphasis} \mathrm{was} \mathrm{placed} \mathrm{on} \mathrm{dopant} \mathrm{seg-}$ regation as a measure to tune the Schottky barrier height and obtain Ohmic contacts. Both methods for dopant segregation: (i) ion implantation into the NiGeSn (Implantation Into Stano Germanide-IISG) and (ii) ion implantation prior to NiGeSn formation (Stano Germanidation Induced Dopant Segregation-SGIDS) were studied. Furthermore, the influence of the Sn content and biaxial compressive strain on dopant segregation was analyzed.

\section{EXPERIMENTAL}

High quality GeSn epilayers with $\mathrm{Sn}$ contents up to $12.5 \% \mathrm{Sn}$ and thicknesses between $40 \mathrm{~nm}$ (pseudomorphic$\mathrm{pm}$ ) and $800 \mathrm{~nm}$ (partially relaxed) were grown on Ge virtual substrates (Ge-VS) on Si (001) wafers using reduced pressure chemical vapor deposition (RPCVD). ${ }^{3,19}$ After native $\mathrm{GeSnO}_{\mathrm{x}}$ removal using a hydrofluoric/hydrochloric acid $(\mathrm{HF} / \mathrm{HCl})$ based wet cleaning solution, ${ }^{20}$ samples were loaded into a sputter-tool and $10 \mathrm{~nm} \mathrm{Ni}$ were deposited by DC sputtering. Thermal treatment in $\mathrm{H}_{2}: \mathrm{N}_{2}$ forming gas at $325^{\circ} \mathrm{C}$ for $10 \mathrm{~s}$ led to the formation of $23 \mathrm{~nm}$ thick metallic $\mathrm{NiGeSn}$ poly-crystalline layers with low contact resistivity. ${ }^{14}$

\section{A. Structural characterization}

XRD $\theta-2 \theta$ scans of $\mathrm{Ni}_{\mathrm{y}}\left(\mathrm{Ge}_{1-\mathrm{x}} \mathrm{Sn}_{\mathrm{x}}\right)_{\mathrm{z}}$ fabricated on several GeSn alloys with different $\mathrm{Sn}$ contents ranging from 0 at. \% $\mathrm{Sn}$ (Ge-VS) to 12.5 at. \% $\mathrm{Sn}$ are shown in Fig. 1(a). The GeSn layers were partially relaxed, with thicknesses of $410 \mathrm{~nm}$ for the 12.5 at. \% Sn layer and $\sim 800 \mathrm{~nm}$ for the other three $\mathrm{Sn}$ contents probed, respectively. The XRD structural analysis revealed the co-existence of several $\mathrm{Ni}_{\mathrm{y}}\left(\mathrm{Ge}_{1-\mathrm{x}} \mathrm{Sn}_{\mathrm{x}}\right)_{\mathrm{z}}$ phases, in agreement with previous studies of NiGeSn formation on pseudomorphically grown (i.e., thin) GeSn layers that revealed mainly $\mathrm{Ni}_{1}\left(\mathrm{Ge}_{1-\mathrm{x}} \mathrm{Sn}_{\mathrm{x}}\right)_{1}$ (Refs. 16 and 18) but also $\mathrm{Ni}_{5}\left(\mathrm{Ge}_{1-\mathrm{x}} \mathrm{Sn}_{\mathrm{x}}\right)_{3}$. ${ }^{14,17}$ This study indicates that for thick (partially relaxed) layers the phase composition changes with the Sn content even though the metallization was performed at the same temperature for all samples $\left(325^{\circ} \mathrm{C}\right)$. For low $\mathrm{Sn}$ content alloys, the main phase is orthorhombic $\mathrm{Ni}_{1}\left(\mathrm{Ge}_{1-\mathrm{x}} \mathrm{Sn}_{\mathrm{x}}\right)_{1}$. At about 8.5 at. \% $\mathrm{Sn}$, the out-of-plane oriented hexagonal $\mathrm{Ni}_{5}\left(\mathrm{Ge}_{1-\mathrm{x}} \mathrm{Sn}_{\mathrm{x}}\right)_{3}$ phase appears and becomes dominant as the Sn content becomes higher and higher. For
NiGeSn alloyed from partially relaxed $\mathrm{Ge}_{0.875} \mathrm{Sn}_{0.125}$ alloys, the well-oriented $\mathrm{Ni}_{5}\left(\mathrm{Ge}_{0.875} \mathrm{Sn}_{0.125}\right)_{3}$ phase is the only one observed. This finding is supported by the fast Fourier transform (FFT) analysis of a $\mathrm{Ni}_{\mathrm{y}}\left(\mathrm{Ge}_{0.92} \mathrm{Sn}_{0.08}\right)_{\mathrm{z}}$ region in the cross-section TEM (XTEM) of Fig. 1(b) showing a hexagonal pattern similar to that reported for $\mathrm{Ni}_{5}(\mathrm{SiGe})_{3}{ }^{21}$ For intermediate $\mathrm{Sn}$ concentrations (i.e., 5.5 at. \% Sn-10 at. \% $\mathrm{Sn}$ ), a third phase with a peak at $47.5^{\circ}$ is also detected. This reflection may be attributed to the Ni-rich phases $\mathrm{Ni}_{2}\left(\mathrm{Ge}_{1-}\right.$ $\left.{ }_{\mathrm{x}} \mathrm{Sn}_{\mathrm{x}}\right)_{1}$ or $\mathrm{Ni}_{3}\left(\mathrm{Ge}_{1-\mathrm{x}} \mathrm{Sn}_{\mathrm{x}}\right)_{2}$. However, due to the multiplicity of possible phases a full crystallographic analysis of NiGeSn is very challenging and beyond the scope of this work.

\section{B. Extraction of sheet resistance and Schottky barrier heights}

The NiGeSn sheet resistance was obtained using the Van der Pauw method. According to Fig. 2, the sheet resistance of NiGeSn is nearly independent of the $\mathrm{Sn}$ content of the GeSn layer. The measured value of about $10-15 \Omega / \square$ is comparable to literature reports for $\mathrm{NiGe} .^{22,23}$

Apart from the sheet resistance, the Schottky barrier height is an important characteristic for the NiGeSn/GeSn interface. It should indeed be as small as possible to allow for low resistive transport through the junction. The analysis of the temperature dependence of metal/semiconductor/ metal (MSM) diode current voltage (I-V) characteristics is

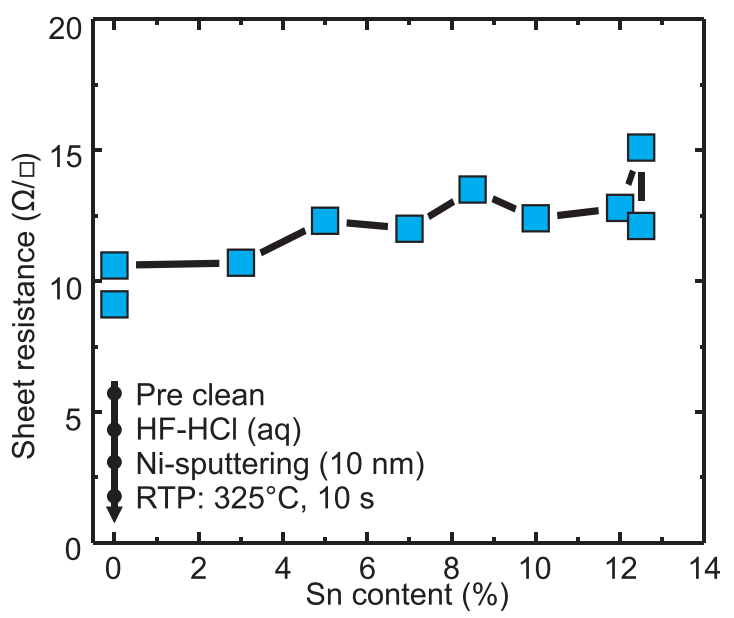

FIG. 2. Sheet resistance of NiGeSn fabricated on GeSn with different Sn contents. The key fabrication steps are shown in the inset. 
reported to be a suitable method allowing the extraction of even very low Schottky barrier heights $(\mathrm{SBH}){ }^{24} \mathrm{~A}$ further advantage of this method is that the effective contact area does not need to be known ruling out possible inaccuracies due to crowding effects. ${ }^{25}$ Thus $\mathrm{NiGeSn} / \mathrm{GeSn} / \mathrm{NiGeSn}$ MSM-diodes have been fabricated using standard CMOS technology including optical lithography and dry-etching.

The I-V characteristics of an exemplary 7\% Sn MSM diode are plotted in Fig. 3(a). Based on these data, the temperature dependence of the current is extracted for certain voltages. According to thermionic emission-diffusion theory, the slope of the declining linear region of the junction current $I$ plotted as $\ln \left(|I| / T^{2}\right)$ vs $1 / T$ [see Fig. 3(b)] provides the $\mathrm{SBH}(\mathrm{V})$ that, due to static and image force lowering, depends linearly on the applied bias: ${ }^{26} \mathrm{SBH}(\mathrm{V})=\mathrm{SBH}_{0}+\beta \mathrm{V}$. Outside the linear region of Fig. 3(b), electrical characteristics are dominated by series and shunt-resistances rather than by the Schottky junction. The $\mathrm{SBH}$ at zero bias, $\mathrm{SBH}_{0}$ (in the following just denoted as $\mathrm{SBH}$ ), is calculated by plotting the voltage dependence of $\mathrm{SBH}(\mathrm{V})[\mathrm{Fig} .3(\mathrm{c})]$ and extrapolating it to $0 \mathrm{~V}$. This method yields the Schottky barrier of the majority carriers as the transport through the junction is mediated by majority carriers. Also, the current that flows in a back-to-back MSM diode is controlled by the lowest Schottky barrier. In an ideal case, both electron and hole $\mathrm{SBH}$ add up to the band gap $\mathrm{E}_{\mathrm{g}}=\mathrm{eSBH}+\mathrm{hSBH}$, providing an easy method for calculating the complementary SBH. However, this equation is not valid if quantum mechanical tunneling through the barrier or minority carrier injection significantly contributes to the carrier transport. ${ }^{25}$ In these cases, only a smaller apparent (effective) SBH can be extracted and eSBH and hSBH do not add up to $\mathrm{E}_{\mathrm{g}}$. The tunneling contribution depends on doping and effective carrier mass. ${ }^{25}$ Increased doping results in a steeper decay of the band bending and thereby in a thinner tunneling barrier whereupon a smaller effective mass allows easier tunneling through the barrier. In the case of Si-Au contacts, the tunneling current exceeds the thermionic current for $\mathrm{N} \geq 10^{18} 1 /$ $\mathrm{cm}^{3}$ at $300 \mathrm{~K} .{ }^{27}$ Since electron mass $\mathrm{m}_{\Gamma}$ and light hole effective mass $\mathrm{m}_{\mathrm{lh}}$ are smaller in $\mathrm{GeSn} \quad\left(\mathrm{m}_{\Gamma} \approx 0.03 \mathrm{~m}_{\mathrm{e}}\right.$, $\left.\mathrm{m}_{\mathrm{lh}} \approx 0.035 \mathrm{~m}_{\mathrm{e}}\right)^{28}$ than in $\mathrm{Si}$, for GeSn tunneling is expected to affect the effective SBH already for significantly smaller doping levels.
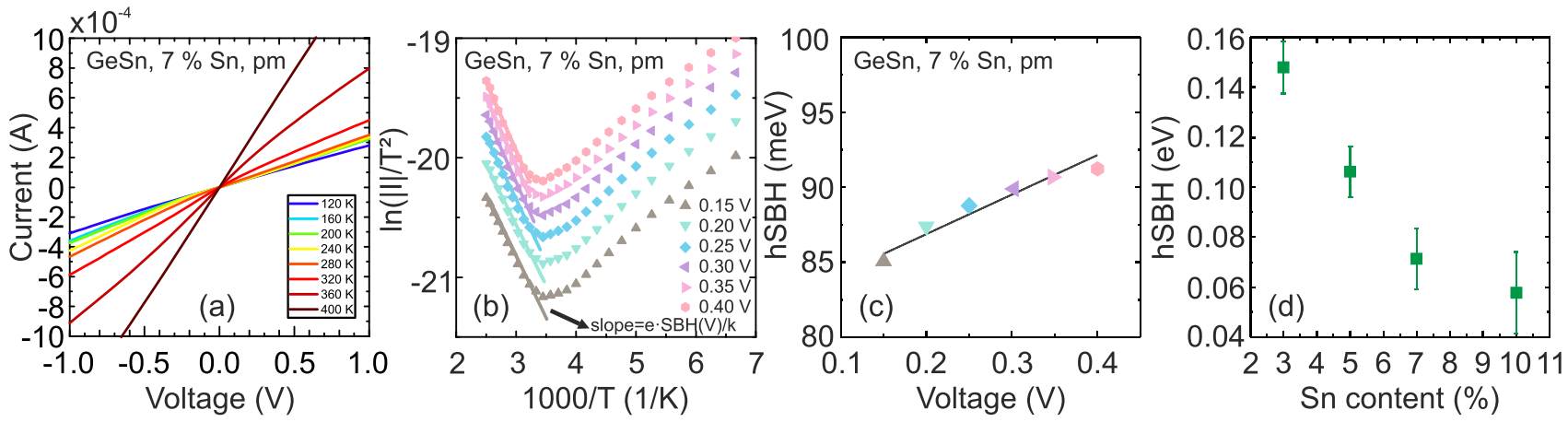

FIG. 3. (a) I-V characteristics of MSM diodes for different temperatures. (b) Corresponding Arrhenius plot of MSM-diode characteristics. e is the elementary charge and $\mathrm{k}$ is the Boltzmann constant. (c) The SBH(V) is extracted from the slope in (b) for different voltages and plotted against the applied voltage. The extracted $\mathrm{hSBH}$ at $0 \mathrm{~V}$ is plotted in (d) against the Sn content. A systematic decrease of the hSBH with the Sn content can be observed.
Another effect that might become relevant for this low bandgap material and affect the effective SBH is minority carrier injection. For Si-Au Schottky diodes, the ratio of minority carrier injection and majority carrier injection $\gamma$ is in the $10^{-5}$ range for low bias and minority carrier injection can be neglected. ${ }^{25}$ However, $\gamma$ quadratically depends on the intrinsic carrier concentration $n_{i}$ which in turn exponentially depends on the bandgap. $\mathrm{n}_{\mathrm{i}}$ is in the $10^{13}$ to mid $10^{14} 1 / \mathrm{cm}^{3}$ range for $\mathrm{GeSn}$ compared to $10^{10} 1 / \mathrm{cm}^{3}$ for $\mathrm{Si}$. As a consequence, minority carrier transport can become relevant for Ge and GeSn as mentioned by Green and Shewchun. ${ }^{29}$ Recently, we experimentally demonstrated that minority carrier response in GeSn MOS-structures is significantly stronger than in $\mathrm{Ge}^{30}$

According to the above discussion, it should be pointed out that the following SBH-values always denote the effective SBH. This way, the hSBH was extracted for several intrinsically p-type $\mathrm{GeSn}$ alloys with $\mathrm{Sn}$ contents ranging from 3 at. $\%$ to 10 at. \%. Small hSBH was extracted for all analyzed Sn-contents in line with recent data on the related $\mathrm{Ni}(\mathrm{SiGeSn}) / \mathrm{p}-\mathrm{SiGeSn}$ system of $\mathrm{hSBH}=0.09 \mathrm{eV}$ for $\mathrm{Si}_{0.07} \mathrm{Ge}_{0.86} \mathrm{Sn}_{0.07 .}{ }^{31}$ This points towards Fermi-level pinning close to the valence band due to metal induced gap states (MIGS) known from metal-germanide contacts. ${ }^{16,32-34} \mathrm{~A}$ systematic decrease of the hSBH with increasing Sn content was evidenced, which is consistent with the bandgap decrease for increasing Sn content [Fig. 3(d)]. However, this does not necessarily infer that changes in hSBH follow changes in the band-alignment. The intrinsic p-type background doping increases with the Sn content (from mid $10^{16} \mathrm{~cm}^{-3}$ to mid $10^{17} \mathrm{~cm}^{-3}$ ) which might also contribute to the reduction of the effective hSBH.

\section{Contact resistance on in-situ doped GeSn}

Generally, low resistance Ohmic contacts require highly doped (degenerated) semiconductors. A typical method to obtain high doping levels, for instance in the source-drain regions of Si CMOS devices, is ion beam implantation. The low thermal stability of $\mathrm{GeSn}$, limited to about $300^{\circ} \mathrm{C}$ $-350^{\circ} \mathrm{C}$, hinders a defect free re-crystallization or efficient annealing of ion implanted regions, making ion implantation inappropriate for GeSn alloys. ${ }^{35}$ These challenges can be circumvented by in-situ doping. It has been demonstrated that 


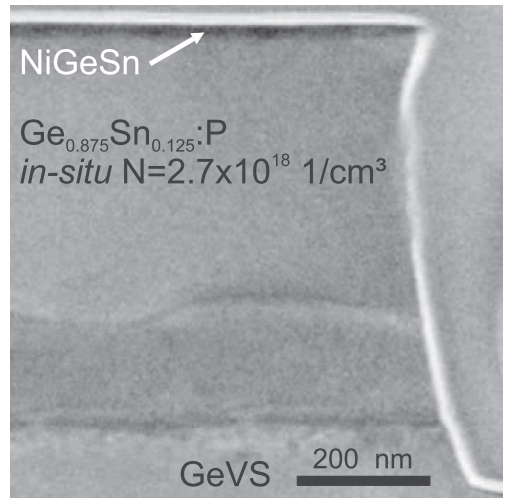

FIG. 4. XTEM image of in-situ phosphorous doped $\mathrm{Ge}_{0.875} \mathrm{Sn}_{0.125}$ with NiGeSn contact showing excellent crystalline quality.

in-situ doping of GeSn is possible and that high doping levels can be achieved for p- and n-type dopants. ${ }^{9,36,37}$ As an example, a nearly defect free NiGeSn on an in-situ phosphorous doped $\mathrm{Ge}_{0.875} \mathrm{Sn}_{0.125}$ layer is shown in the XTEM micrograph of Fig. 4. The $n$-type carrier concentration amounts to $8 \times 10^{18} \mathrm{~cm}^{-3}$. Here, only the typical dislocation half-loops due to strain relaxation are visible at the $\mathrm{GeSn} / \mathrm{Ge}$ interface. However, no threading dislocations or extended crystal defects appear in the TEM lamella. GeSn retains its excellent crystalline quality during metallization annealing. Transfer length model (TLM) structures were then fabricated for contact resistance measurements as follows.

I-V measurements were performed for neighboring contacts with differing distances [see Fig. 5(a)] and the resistance extracted by fitting the linear region. The slope of a linear fit of the measured resistance as a function of the
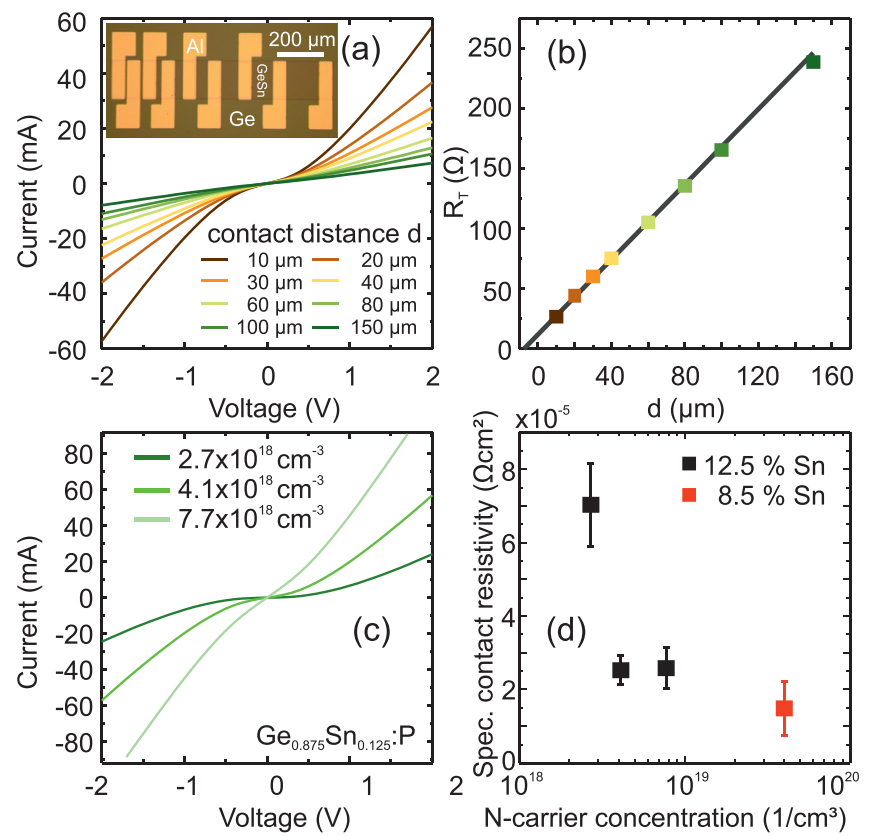

FIG. 5. (a) I-V characteristics of a TLM-structure for different contact distances. The inset shows an optical microscopy image of a fabricated TLMstructure. The resistances extracted from the linear region in (a) are plotted versus the contact distance in (b). (c) I-V characteristics of TLM structures with different phosphorous doping levels in GeSn. (d) Specific contact resistivity vs. doping concentration. contact distance [Fig. 5(b)] is linked to the sheet resistance $\mathrm{R}_{\mathrm{sq}}$ of the GeSn:P layer, whereas half the $\mathrm{x}$-intercept gives the transfer length $\mathrm{L}_{\mathrm{T}}$. This way, the specific contact resistivity $\rho_{\mathrm{c}}=\mathrm{L}_{\mathrm{T}} * \mathrm{R}_{\mathrm{sq}}{ }^{2}$ was extracted for different doping levels and $\mathrm{Sn}$ contents. Figure 5(c) shows the I-V characteristics between two contacts for in-situ doped $n$-type $\mathrm{Ge}_{0.875} \mathrm{Sn}_{0.125}$ with three different doping levels. The behavior becomes Ohmic for higher doping levels, which is consistent with an increased tunneling component through the Schottky barrier as also reported by Srinivasan et al ${ }^{38}$ for metal/n-GeSn contacts. Specific contact resistivities in the low $10^{-5} \Omega \mathrm{cm}^{2}$ range have been achieved [Fig. 5(d)], i.e., significantly lower than those reported by $\mathrm{Li}$ et al. for $\mathrm{NiGeSn} / n-\mathrm{GeSn}$ contacts $\left(\rho_{\mathrm{c}}=6.4 \times 10^{-4} \Omega \cdot \mathrm{cm}^{2}\right)$ and in reasonable agreement with values measured by Han et al. ${ }^{15}$ for $p$-type $\mathrm{NiGeSn} / p$-GeSn contacts. It should be noted that contact resistivities below $10^{-6} \Omega \cdot \mathrm{cm}^{2}$ are required for device production. ${ }^{39}$ The lowest reported values for metal/GeSn contacts are in the $10^{-6}$ $\Omega \cdot \mathrm{cm}^{2}$ range. ${ }^{38}$

\section{Dopant segregation in NiGeSn}

The process flow for the SGIDS and IISG dopant segregation schemes in NiGeSn/GeSn contacts is shown in Figs. 6(a)-6(d) and Figs. 6(a) and 6(e)-6(h), respectively. In both approaches, standard cleanroom technology is used for device fabrication. First, the active area is defined by photo lithography and dry etching. Mesa passivation is realized with a stack of $10 \mathrm{~nm}$ atomic layer deposited $\mathrm{Al}_{2} \mathrm{O}_{3}$ followed by $150 \mathrm{~nm}$ of plasma enhanced chemical vapor deposited $\mathrm{SiO}_{2}$. Contact windows are then opened with dry-etching [Fig. 6(a)].

First, we analyze DS using the IISG technique [see flow Figs. 6(a) and 6(e)-6(h)]. After converting $10 \mathrm{~nm} \mathrm{Ni}$ to $\sim 23 \mathrm{~nm}$ thick NiGeSn by annealing at $325^{\circ} \mathrm{C}$ for $10 \mathrm{~s}$ [Figs. 6(e) and 6(f)], $p$-type $\left(\mathrm{BF}_{2}{ }^{+}, 15.8 \mathrm{keV}\right)$ or $n$-type dopants $\left(\mathrm{P}^{+}, 10 \mathrm{keV}\right.$, or $\left.\mathrm{As}^{+}, 13 \mathrm{keV}\right)$ with a dose of $1 \times 10^{15} \mathrm{~cm}^{-3}$ were implanted into the metallic NiGeSn layers [Fig. 6(g)]. The dopant implantation energy was chosen such that the dopant distribution resides completely in the NiGeSn layer to avoid crystal damage of the underlying GeSn layer. The implantation was followed by a second annealing at $325^{\circ} \mathrm{C}$ for $10 \mathrm{~s}$ [Fig. 6(h)] to drive out the dopants to the NiGeSn/ GeSn interface.

The elemental distribution was analyzed by time of flight secondary ion mass spectroscopy (ToF-SIMS) and showed no dopant pile-up at the $\mathrm{NiGeSn} / \mathrm{GeSn}$ interface independent of the dopant species [Figs. 7(a)-7(c)]. Consequently, dopant segregation by ion implantation into $\mathrm{NiGeSn}$ (IISG) is not effective given the limited annealing temperature allowed by the GeSn stability and the choice of dopant. The diffusion of dopants from the NiGeSn towards the NiGeSn/GeSn interface is obviously not high enough for a significant interface pile-up effect.

\section{E. Stanogermanidation induced dopant segregation (SGIDS)}

As an alternate approach, the same ion species, $\mathrm{BF}_{2}{ }^{+}$, $\mathrm{P}^{+}$, and $\mathrm{As}^{+}$, were tested for SGIDS. The corresponding 
(a)

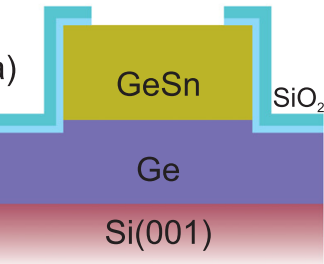

Contact window definition (b)
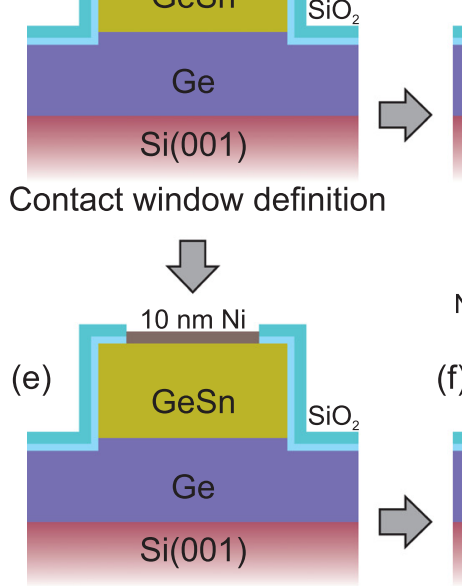

Ni deposition

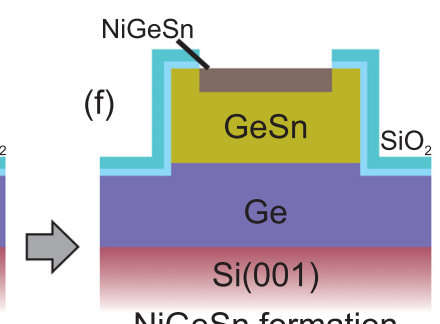

NiGeSn formation

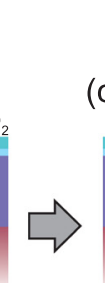

(c)

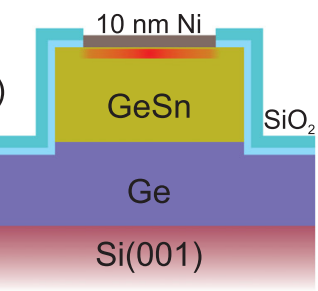

Ni deposition

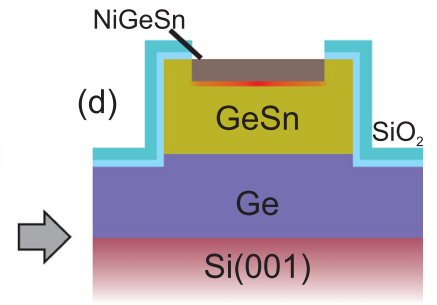

DS anneal

FIG. 6. Fabrication procedure for stanogermanidation induced dopant segregation (SGIDS) (a)-(d) and for dopant segregation by implantation into nickelstanogermanide (IISG) (a) and (e)-(h).

process flow is depicted in Figs. 6(a-(d). Here, the implantation occurred directly into the GeSn using the following implantation parameters [Fig. 6(b)]: $10 \mathrm{keV}$ for $\mathrm{BF}_{2}{ }^{+}$, $7 \mathrm{keV}$ for $\mathrm{P}^{+}$, and $13 \mathrm{keV}$ for $\mathrm{As}^{+}$at a dose of $1 \times 10^{15}$ $\mathrm{cm}^{-2}$. After Ni deposition [Fig. 6(c)], NiGeSn was formed and dopants were driven out in one step thanks to annealing at $325^{\circ} \mathrm{C}$ [Fig. 6(d)]. The dopant ion implantation energy was such that the implantation induced damaged region was fully consumed by the NiGeSn, while the dopants may diffuse to the interface with the crystalline (i.e., undamaged) GeSn below. In contrast to the IISG case, the ToFSIMS spectra [Figs. 7(d) and (f)] indicate a clear dopant distribution peak at the metal/semiconductor interface for
As and B dopants, whereas P shows no dopant segregation effect [Fig. 7(e)].

In opposition to our findings, $\mathrm{P}$ segregation during NiGe formation was reported in Ref. 22 albeit at a higher formation temperature of $400^{\circ} \mathrm{C}$. In addition, it was found less effective than As DS. ${ }^{22}$ The formation of NiGeSn is connected with significant volume changes and the creation of point defects that can enhance dopant diffusion ${ }^{40}$ at the evolving $\mathrm{NiGeSn} / \mathrm{GeSn}$ interface even at low temperatures leading to a snow plow effect. This might explain why SGIDS worked but not IISG.

In order to gain a deeper understanding of the parameters influencing the SGIDS, a systematic study is presented
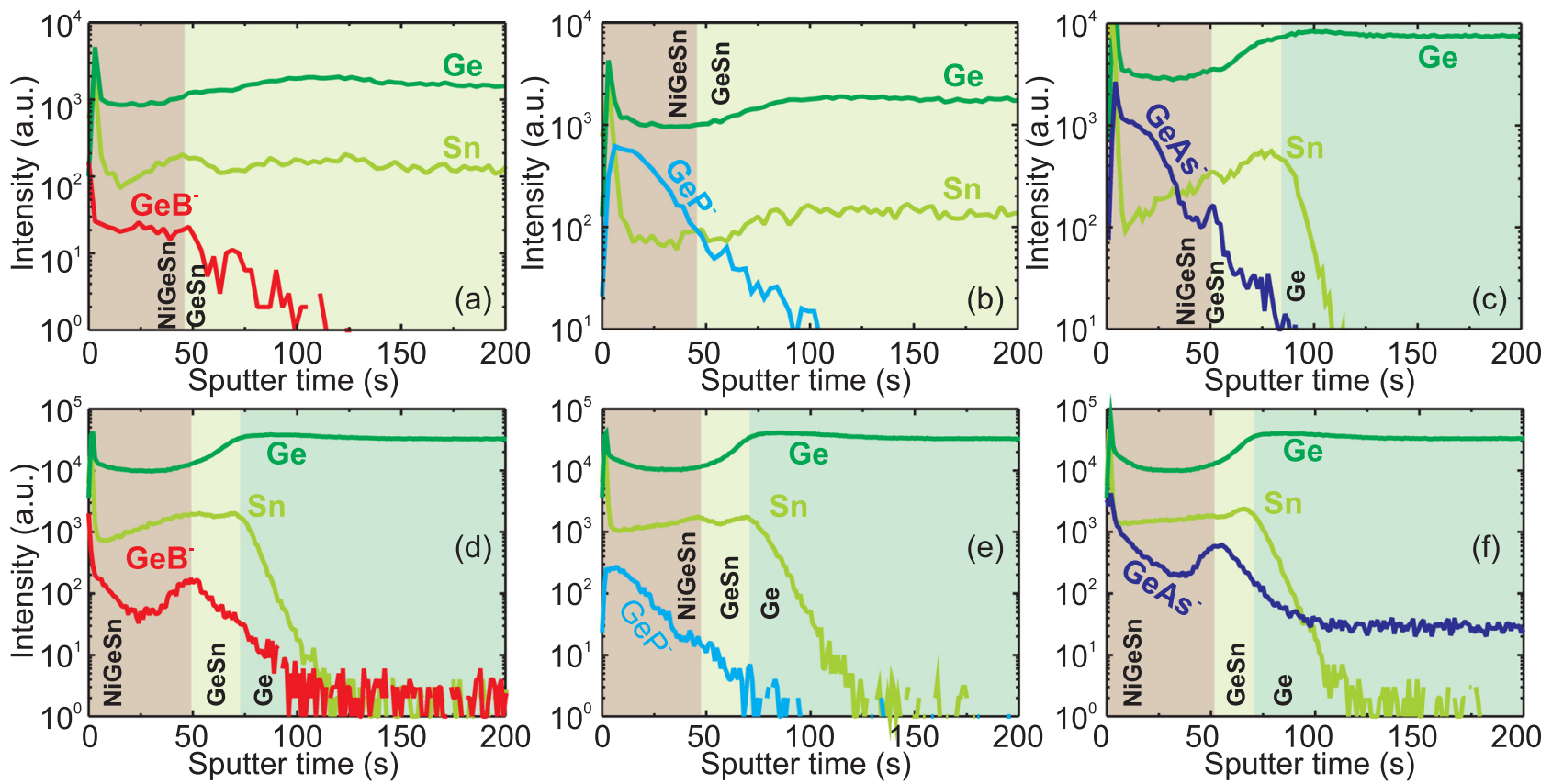

FIG. 7. Elemental TOF-SIMS spectra at the NiGeSn/GeSn interface for IISG (a)-(c) and SGIDS (d)-(f). No DS is observed for IISG for B, P, or As (a)-(c). However, SGIDS is observed for As and $\mathrm{BF}_{2}$ (d) and (f) implant, not for $\mathrm{P}$ (e). 


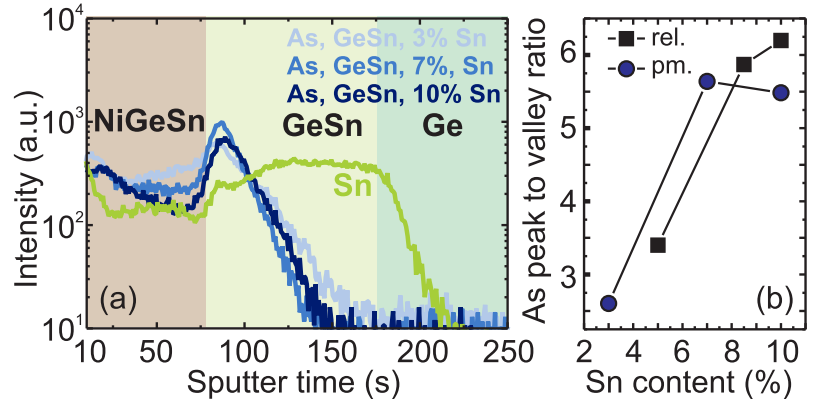

FIG. 8. (a) Elemental TOF-SIMS spectra of SGIDS with arsenic on pseudomorphically (pm.) grown GeSn with different Sn contents. For a better detection, $\mathrm{GeAs}^{-}$is used as the As signal here. (b) Peak to valley ratio of the As concentration $v s$. Sn content for SGIDS on pseudomorphic GeSn and relaxed (rel.) GeSn. The Sn-signal is exemplarily shown for the 7\% $\mathrm{Sn}$ sample.

below for B and As dopants for various Sn contents and elastic strains in the GeSn layer. Samples ranging from $0 \% \mathrm{Sn}$ (Ge-VS) up to $12 \% \mathrm{Sn}$ and from pseudomorphically grown on Ge (fully strained) to partially relaxed layers were analyzed.

It should be noted that the complexity of the DS-process only allows a qualitative discussion of the parameters influencing the dopant redistribution. ${ }^{40}$

Dopant segregation of As at the $\mathrm{NiGeSn} / \mathrm{GeSn}$ interface becomes more pronounced as the Sn content increases, for both pseudomorphic and partially relaxed layers, as shown in Fig. 8(a). This is obvious when looking at the peak-to-valley ratio of the dopant signal in the NiGeSn layer as shown in Fig. 8(b): it increases by more than a factor of two when going from 3 at.\% Sn to 10 at.\% Sn in the GeSn layer. It is known that the number of point defects (e.g., vacancies) in GeSn increases with the Sn content. ${ }^{41}$ Since As diffuses via vacancies in $\mathrm{Ge}^{42}$ (and thus probably also in $\mathrm{GeSn}$ ), this might result in stronger diffusion of As explaining the enhanced SGIDS ${ }^{43}$ effect.

Interestingly, SGIDS with B in NiGeSn shows the opposite Sn dependence, as seen in Fig. 9. The peak to valley ratio of the $\mathrm{B}$ concentration drops by a factor of roughly three when going from $3 \%$ to $10 \% \mathrm{Sn}$. B diffuses interstitially and thereby relies on a fundamentally different mechanism than As diffusion. ${ }^{42}$ However, the observed changes in the doping profile are mainly within the NiGeSn layer.

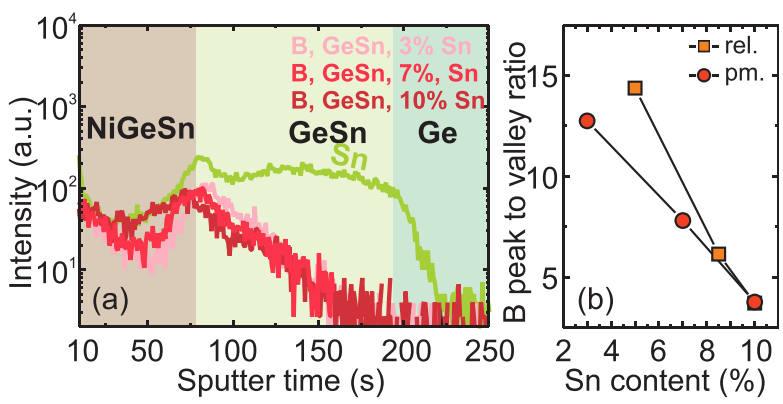

FIG. 9. (a) ToF-SIMS analysis of SGIDS with boron on pseudomorphically grown GeSn layers with different $\mathrm{Sn}$ contents. $\mathrm{GeB}^{-}$is used as the B signal. (b) Peak to valley ratio of the B concentration vs. Sn content for SGIDS on pseudomorphic (pm.) and relaxed (rel.) GeSn alloys. The Sn-signal is exemplarily shown for the $7 \%$ Sn sample.
Following the discussion by Murarka and Williams, ${ }^{40}$ the increased B signal in the NiGeSn-layer might be attributed to an enhanced solid solubility of B in NiGeSn with increasing Sn-content. At the same time, achieving high B doping levels in GeSn is more challenging than in Ge, presumably due to a decreased solid solubility of B in GeSn. ${ }^{37}$ The interplay of both effects results in a reduction of the segregation coefficient (peak to valley ratio) as the Sn content increases.

\section{Strain dependence}

It is known that the diffusion of dopants depends on the strain and the existence of point-defects. We have thus quantified the dependence of SGIDS on biaxial compressive lattice strain in the GeSn layer. Thin GeSn epilayers grown pseudomorphically on Ge are highly compressively strained. When growing GeSn beyond a certain critical thickness (which is a function of the Sn content), these layers start to plastically relax the built-in compressive strain via the formation of numerous misfit dislocations. $\mathrm{Ge}_{0.875} \mathrm{Sn}_{0.125}$ layers with thicknesses of 90, 280, and $414 \mathrm{~nm}$ were used here, corresponding to compressive strains of $-1.56 \%,-0.48 \%$, and $-0.43 \%$, respectively. Again doped NiGeSn layers with $\mathrm{As}^{+}$ and $\mathrm{BF}_{2}{ }^{+}$ion implantation were formed by SGIDS. For As atoms, the dopant segregation peak becomes less pronounced as the GeSn thickness increases (the compressive strain decreases) (Fig. 10). As vacancy diffuser, ${ }^{42}$ large volume atoms like As are expected to show enhanced diffusion under compressive strain. ${ }^{44}$ In contrast, no strain dependence was observed for SGIDS with boron. Since boron diffuses via interstitials, less strain dependence was expected.

\section{F. Schottky barrier height modulation with dopant segregation}

In the following, the effect of DS on the electrical characteristics of MSM diodes is investigated. Thus, MSM diodes on in-situ doped $\mathrm{Ge}_{0.875} \mathrm{Sn}_{0.125}: \mathrm{P}$ were fabricated using the process scheme for SGIDS described above. Whereas the un-implanted sample shows a slight diode-like behavior, the I-V characteristics of the sample with As dopant segregation (complementary doping) are perfectly Ohmic [Fig. 11(a)]. In contrast, the sample with boron dopant segregation (counter doping) shows as expected an enhanced diode behavior. The corresponding Schottky

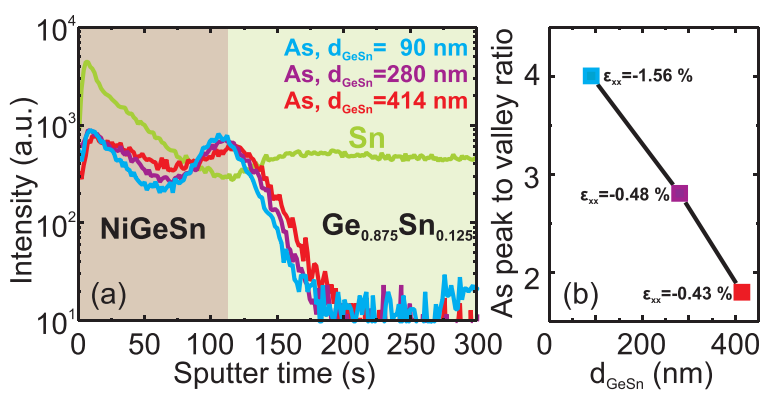

FIG. 10. (a) ToF-SIMS analysis for SGIDS with arsenic on $\mathrm{Ge}_{0.875} \mathrm{Sn}_{0.125}$ with different $\mathrm{GeSn}$ thicknesses. For better detection $\mathrm{GeAs}^{-}$was used as the As signal. The Sn signal is taken for the $414 \mathrm{~nm}$ sample. (b) Peak to valley ratio of the As concentration. 


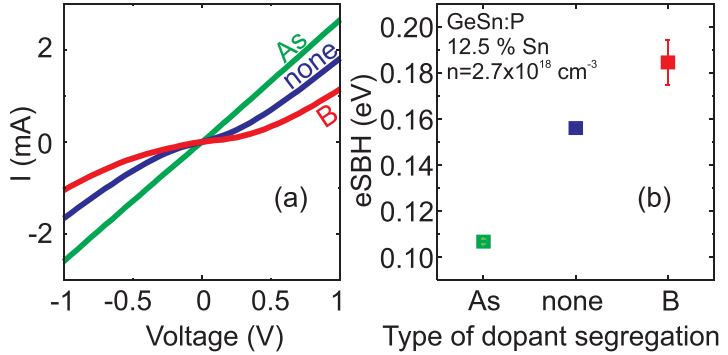

FIG. 11. (a) I-V characteristics of $\mathrm{NiGeSn} / \mathrm{Ge}_{0.875} \mathrm{Sn}_{0.125}: \mathrm{P} \mathrm{MSM}$ diodes with As and B segregation and (b) corresponding eSBH.

barriers are shown in Fig. 11(b). Arsenic dopant segregation reduces the Schottky barrier, whereas boron dopant segregation leads to an increased Schottky barrier in this in-situ ndoped layer. The observed eSBH is comparable to that observed by Tong et al. ${ }^{16}$ for $\mathrm{S}$ and Se segregation on $\mathrm{NiGeSn} / \mathrm{n}-\mathrm{GeSn}$ contacts. What is striking though is the fact that even the eSBH without dopant segregation is comparably low since one might expect an eSBH close to $\mathrm{E}_{\mathrm{g}}$ considering the fact that extremely small hSBH were observed on pGeSn [Fig. 3(d)]. However in compliance with the discussion in Section II B one expects a significant contribution from tunneling for the doping level of $2.7 \times 10^{18} 1 / \mathrm{cm}^{3}$ which effectively reduces the apparent eSBH.

\section{CONCLUSION}

We have presented a comprehensive study of NiGeSn contacts on GeSn with a wide range of $\mathrm{Sn}$ contents for GeSn-based electronic devices. A low NiGeSn sheet resistance was obtained for the whole Sn content range probed. TEM and XRD analysis of NiGeSn revealed the presence of several NiGeSn polycrystalline phases. Investigations of dopant segregation have shown that dopant segregation can be achieved during NiGeSn formation for both p- and n-type dopants and used for Schottky barrier height tuning. Furthermore, dependencies of dopant segregation in NiGeSn on the Sn content and layer strain have been analyzed. These results are important to obtain low contact resistances in GeSn devices such as MOSFETs or electrically pumped lasers.

\section{ACKNOWLEDGMENTS}

This research received partial funding from the EU FP7 project E2SWITCH (619509) and the BMBF project UltraLowPow (16ES0060 K).

${ }^{1}$ S. Wirths, D. Buca, and S. Mantl, Prog. Cryst. Growth Charact. Mater. 62, 1 (2016).

${ }^{2}$ S. Wirths, R. Geiger, N. von den Driesch, G. Mussler, T. Stoica, S. Mantl, Z. Ikonic, M. Luysberg, S. Chiussi, J. M. Hartmann, H. Sigg, J. Faist, D. Buca, and D. Grützmacher, Nat. Photonics 9, 88 (2015).

${ }^{3}$ N. von den Driesch, D. Stange, S. Wirths, G. Mussler, B. Holländer, Z. Ikonic, J. M. Hartmann, T. Stoica, S. Mantl, D. Grützmacher, and D. Buca, Chem. Mater. 27, 4693 (2015).

${ }^{4}$ G. Han, S. Su, L. Wang, W. Wang, X. Gong, Y. Yang, Ivana, P. Guo, C. Guo, G. Zhang, J. Pan, Z. Zhang, C. Xue, B. Cheng, and Y. Yeo, in 2012 Symposium on VLSI Technology (IEEE, 2012), pp. 97-98.
${ }^{5}$ X. Gong, G. Han, F. Bai, S. Su, P. Guo, Y. Yang, R. Cheng, D. Zhang, G. Zhang, C. Xue, B. Cheng, J. Pan, Z. Zhang, E. S. Tok, D. Antoniadis, and Y.-C. Yeo, IEEE Electron Device Lett. 34, 339 (2013).

${ }^{6}$ J. Schulze, A. Blech, A. Datta, I. A. Fischer, D. Hähnel, S. Naasz, E. Rolseth, and E.-M. Tropper, Solid State Electron. 110, 59 (2015).

${ }^{7}$ R. Pandey, C. Schulte-Braucks, R. N. Sajjad, M. Barth, R. K. Ghosh, B. Grisafe, P. Sharma, N. von den Driesch, A. Vohra, B. Rayner, R. Loo, S. Mantl, D. Buca, C.-C. Yeh, C.-H. Wu, W. Tsai, D. Antoniadis, and S. Datta, in 2016 IEEE International Electron Devices Meeting (IEEE, 2016), pp. 19.6.1-19.6.4.

${ }^{8}$ Y. Yang, G. Han, P. Guo, W. Wang, X. Gong, L. Wang, K. L. Low, and Y. Yeo, IEEE Trans. Electron Devices 60, 4048 (2013).

${ }^{9}$ C. Schulte-Braucks, D. Stange, N. von den Driesch, S. Blaeser, Z. Ikonic, J. M. Hartmann, S. Mantl, and D. Buca, Appl. Phys. Lett. 107, 042101 (2015).

${ }^{10}$ Z. Qiu, Z. Zhang, M. Östling, and S. Zhang, IEEE Trans. Electron Devices 55, 396 (2008).

${ }^{11}$ Y. Hacham-Diamand, T. Osaka, M. Datta, and T. Ohba, Advanced Nanoscale ULSI Interconnects: Fundamentals and Applications (Springer, New York, 2009).

${ }^{12}$ T. Grzela, G. Capellini, W. Koczorowski, M. A. Schubert, R. Czajka, N. J. Curson, I. Heidmann, T. Schmidt, J. Falta, and T. Schroeder, Nanotechnology 26, 385701 (2015).

${ }^{13}$ Q. Liu, W. Geilei, Y. Guo, X. Ke, H. Radamson, H. Liu, C. Zhao, and J. Luo, ECS J. Solid State Sci. Technol. 4, P67 (2014).

${ }^{14}$ S. Wirths, R. Troitsch, G. Mussler, J.-M. Hartmann, P. Zaumseil, T. Schroeder, S. Mantl, and D. Buca, Semicond. Sci. Technol. 30, 055003 (2015).

${ }^{15}$ G. Han, S. Su, Q. Zhou, P. Guo, Y. Yang, C. Zhan, L. Wang, W. Wang, Q. Wang, C. Xue, B. Cheng, and Y. Yeo, IEEE Electron Device Lett. 33, 634 (2012).

${ }^{16}$ Y. Tong, G. Han, B. Liu, Y. Yang, L. Wang, W. Wang, and Y.-C. Yeo, IEEE Trans. Electron Devices 60, 746 (2013).

${ }^{17}$ D. Lei, W. Wang, Z. Zhang, J. Pan, X. Gong, G. Liang, E. S. Tok, and Y. C. Yeo, J. Appl. Phys. 119, 024502 (2016).

${ }^{18}$ L. Wang, G. Han, S. Su, Q. Zhou, Y. Yang, P. Guo, W. Wang, Y. Tong, P. S. Y. Lim, B. Liu, E. Y.-J. Kong, C. Xue, Q. Wang, B. Cheng, and Y.-C. Yeo, Electrochem. Solid-State Lett. 15, H179 (2012).

${ }^{19}$ N. von den Driesch, D. Stange, S. Wirths, D. Rainko, I. Povstugar, A. Savenko, U. Breuer, R. Geiger, H. Sigg, Z. Ikonic, J.-M. Hartmann, D. Grützmacher, S. Mantl, and D. Buca, Small 13, 1603321 (2017).

${ }^{20}$ C. Schulte-Braucks, N. von den Driesch, S. Glass, A. T. Tiedemann, U. Breuer, A. Besmehn, J.-M. Hartmann, Z. Ikonic, Q. T. Zhao, S. Mantl, and D. Buca, ACS Appl. Mater. Interfaces 8, 13133 (2016).

${ }^{21}$ L. Liu, L. Jin, L. Knoll, S. Wirths, A. Nichau, D. Buca, G. Mussler, B. Holländer, D. Xu, Z. Feng Di, M. Zhang, Q.-T. Zhao, and S. Mantl, Appl. Phys. Lett. 103, 231909 (2013).

${ }^{22}$ M. Mueller, Q. T. Zhao, C. Urban, C. Sandow, D. Buca, S. Lenk, S. Estévez, and S. Mantl, Mater. Sci. Eng. B 154-155, 168 (2008).

${ }^{23}$ J. Y. Spann, R. A. Anderson, T. J. Thornton, G. Harris, S. G. Thomas, and C. Tracy, IEEE Electron Device Lett. 26, 151 (2005).

${ }^{24}$ E. Dubois and G. Larrieu, J. Appl. Phys. 96, 729 (2004).

${ }^{25}$ S. M. Sze and K. K. Ng, Physics of Semiconductor Devices (Wiley, 2006).

${ }^{26}$ J. M. Andrews and M. P. Lepselter, Solid State Electron. 13, 1011 (1970).

${ }^{27}$ C. Y. Chang and S. M. Sze, Solid State Electron. 13, 727 (1970).

${ }^{28}$ K. Lu Low, Y. Yang, G. Han, W. Fan, and Y.-C. Yeo, J. Appl. Phys. 112, 103715 (2012).

${ }^{29}$ M. A. Green and J. Shewchun, Solid State Electron. 16, 1141 (1973).

${ }^{30}$ C. Schulte-Braucks, K. Narimani, S. Glass, N. von den Driesch, J. M. Hartmann, Z. Ikonic, V. V. Afanas'ev, Q. T. Zhao, S. Mantl, and D. Buca, ACS Appl. Mater. Interfaces 9, 9102 (2017).

${ }^{31}$ J. Zheng, S. Wang, X. Zhang, Z. Liu, C. Xue, C. Li, Y. Zuo, B. Cheng, and Q. Wang, IEEE Electron Device Lett. 36, 878 (2015).

${ }^{32}$ K. Ikeda, Y. Yamashita, N. Sugiyama, N. Taoka, and S. Takagi, Appl. Phys. Lett. 88, 152115 (2006).

${ }^{33}$ A. Agrawal, N. Shukla, K. Ahmed, and S. Datta, Appl. Phys. Lett. 101, 042108 (2012).

${ }^{34}$ S. Zhu, R. Li, S. J. Lee, M. F. Li, A. Du, J. Singh, C. Zhu, A. Chin, and D. L. Kwong, IEEE Electron Device Lett. 26, 81 (2005).

${ }^{35}$ C. Schulte-Braucks, S. Glass, E. Hofmann, D. Stange, N. von den Driesch, J. M. Hartmann, Z. Ikonic, Q. T. Zhao, D. Buca, and S. Mantl, Solid State Electron. 128, 54 (2017).

${ }^{36}$ C. L. Senaratne, J. D. Gallagher, T. Aoki, J. Kouvetakis, and J. Menéndez, Chem. Mater. 26, 6033 (2014). 
${ }^{37}$ B. Vincent, F. Gencarelli, H. Bender, C. Merckling, B. Douhard, D. H Petersen, O. Hansen, H. H. Henrichsen, J. Meersschaut, W. Vandervorst, M. Heyns, R. Loo, and M. Caymax, Appl. Phys. Lett. 99, 152103 (2011).

${ }^{38}$ V. S. S. Srinivasan, I. A. Fischer, L. Augel, A. Hornung, R. Koerner, K. Kostecki, M. Oehme, E. Rolseth, and J. Schulze, Semicond. Sci. Technol. 31, 08LT01 (2016).

${ }^{39}$ D. K. Schroder, Semiconductor Material and Device Characterization (Wiley, 2006).
${ }^{40}$ S. P. Murarka, J. Vac. Sci. Technol., B: Microelectron. Nanometer Struct. 5, 1674 (1987).

${ }^{41}$ E. Kamiyama, S. Nakagawa, K. Sueoka, T. Ohmura, T. Asano, O. Nakatsuka, N. Taoka, S. Zaima, K. Izunome, and K. Kashima, Appl. Phys. Express 7, 021302 (2014).

${ }^{42}$ H. Bracht and S. Brotzmann, Mater. Sci. Semicond. Process. 9, 471 (2006).

${ }^{43}$ M. Wittmer and K. N. Tu, Phys. Rev. B 29, 2010 (1984).

${ }^{44}$ S. T. Dunham, M. Diebel, C. Ahn, and C. L. Shih, J. Vac. Sci. Technol., B: Microelectron. Nanometer Struct. 24, 456 (2006). 